\title{
An Explicit Time-Domain Hybrid Formulation Based on the Unified Boundary Condition
}

Niel Madsen, Benjamin Fasenfest, Daniel White, Mark Stowell, Vikram Jandhyala, James Pingenot, Nathan Champagne, John Rockway

March 4, 2007 
This document was prepared as an account of work sponsored by an agency of the United States Government. Neither the United States Government nor the University of California nor any of their employees, makes any warranty, express or implied, or assumes any legal liability or responsibility for the accuracy, completeness, or usefulness of any information, apparatus, product, or process disclosed, or represents that its use would not infringe privately owned rights. Reference herein to any specific commercial product, process, or service by trade name, trademark, manufacturer, or otherwise, does not necessarily constitute or imply its endorsement, recommendation, or favoring by the United States Government or the University of California. The views and opinions of authors expressed herein do not necessarily state or reflect those of the United States Government or the University of California, and shall not be used for advertising or product endorsement purposes.

This work was performed under the auspices of the U.S. Department of Energy by University of California, Lawrence Livermore National Laboratory under Contract W-7405-Eng-48. 


\title{
An Explicit Time-Domain Hybrid Formulation Based on the Unified Boundary Condition
}

\author{
Niel Madsen, Benjamin J. Fasenfest, Daniel White, Mark Stowell, Vikram Jandhyala, James Pingenot, \\ Nathan J. Champagne and John D. Rockway
}

\begin{abstract}
An approach to stabilize the two-surface, time domain FEM/BI hybrid by means of a unified boundary condition is presented. The first-order symplectic finite element formulation [1] is used along with a version of the unified boundary condition of Jin [2] reformulated for Maxwell's firstorder equations in time to provide both stability and accuracy over the first-order ABC. Several results are presented to validate the numerical solutions. In particular the dipole in a free-space box is analyzed and compared to the Dirchlet boundary condition of Ziolkowski and Madsen [3] and to a Neuman boundary condition approach.
\end{abstract}

Index Terms - time domain, integral equations, hybrid formulation, finite element method, method of moments.

\section{INTRODUCTION}

$\mathrm{H}$ YBRID solutions to time-domain electromagnetic problems offer many advantages when solving open-region scattering or radiation problems. These formulations can take advantage of finite-element or finite-difference volume discretization for the features of interest, allowing for intricate features with inhomogeneous materials. This volume region can then be bounded by a layer of planar boundary elements to form a radiating boundary condition, as depicted in Figure 1. In most time-domain hybrid formulations, two separate surfaces are used to set the boundary condition; an inner layer where equivalent currents are formed, and an outer layer where the fields are evaluated and used to truncate the volume mesh. This concept has been implemented previously, using the boundary elements to set the E-field, H-field, or both for an FDTD grid, for example in [3][4][5], and as a mixed boundary condition for the second-order wave equation solved by finite elements [4]. Further study has focused on using fast methods, such as the Plane Wave Time Domain method [2][5] or FFT-based methods [6] to accelerate the BEM calculations. In addition, single-surface hybrids which fuse the same surface to produce currents and satisfy fields have been succesfull when coupled with an implicit finite element solver [6].

The focus of this paper is the long-term stability of the twosurface hybrid solver. The stability within the finite element region is maintained by solving Maxwell's coupled first-order equations for the electric and magnetic fields with an explicit, symplectic time stepper. However, to guarantee late-time stability, the accuracy and stability of the radiation boundary condition used to truncate the finite element mesh must also be ensured. The two-surface boundary integral approach for FDTD is can be implemented by applying the computed fields to the outer boundary as either a Dirchlet boundary condition (based on the electric field) [3] or a Neumann boundary condition (based on the magnetic field) [4]. However, these methods have late-time instabilities when applied to finite element hybrids. The temperament of the finite element region can include interior resonances and have spurious highfrequency or DC effects. These create numerical errors which can build-up over time and destabilize the boundary element solution. While accurate in the short-term, a hybrid solver utilizing these RBC methods will eventually go unstable for some problems.

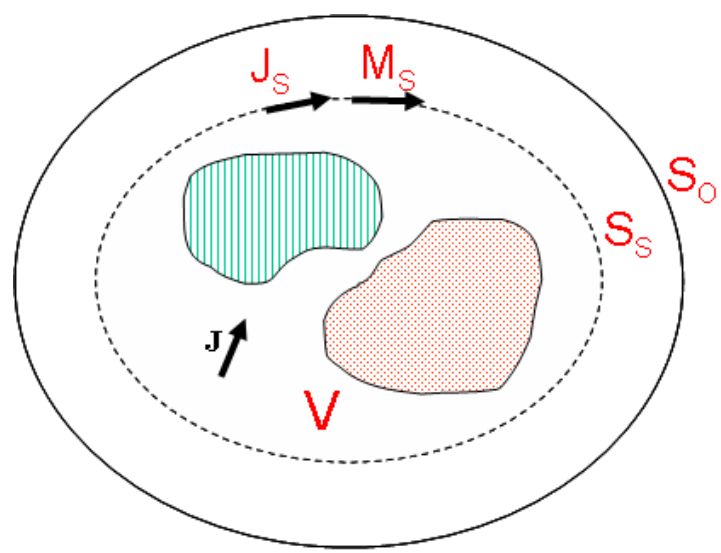

Fig. 1: The geometry of the finite element and method of moment two-surface hybrid. The finite element region is truncated with a radiating surface $S_{0}$. Interior to this surface within the volume is a specified source surface $S_{s}$ on which equivalent surface currents $\boldsymbol{J}(\mathbf{r}, t)$ and $\boldsymbol{M}(\mathbf{r}, t)$ are computed due to the interior electric and magnetic fields.

Instead, a Unified Boundary Condition (UBC) is used here as a means of eliminating errors in the solution to provide long-term stability. Similar to the method used in [2], the UBC is designed as an accurate radiation "correction" to the first-order ABC. It effectively combines both the electric and magnetic field, which combats the cascading instabilities (e.g., interior resonances, numerical dispersion) within the finite element solution. The UBC is more accurate than the first- 
order $\mathrm{ABC}$ for radiation problems. More importantly, it remains stable over longer times when compared to the other hybrid RBC methods for the same mesh.

This paper is organized in the following manner. In Section II, the explicit time domain formulation for the finite element and method of moment hybrid is presented. The details of the unified boundary condition for accurate truncation of the volume mesh is also detailed. A numerical procedure to solve the corresponding explicit equations is presented in Section III. In Section IV, numerical results are presented to validate the proposed method. Finally, conclusions about the method are given in the section $\mathrm{V}$.

\section{AN EXPLICIT TIME-DOMAIN HYBRID FORMULATION}

In this section, at time-domain hybrid to solve Maxwell's first-order equations is formulated. The electric and magnetic fields are determined from Maxwell's coupled first-order equations evaluated within the arbitrary-shaped volumetric domain V. To provide an accurate radiation boundary condition for the fields, a two surface hybrid is proposed. The fields from the interior of the problem excite equivalent surface currents on a source surface, $\mathrm{S}_{\mathrm{s}}$. These sources radiate outward between the two surfaces using a mixed-potential integral equation formulation similar to [7] and are used to compute either the electric field, magnetic field, or both on the outer surface $\mathrm{S}_{0}$. This provides an accurate radiation boundary condition for the original interior $\mathrm{E}$ and $\mathrm{B}$ fields.

To begin, the solution for the electric and magnetic fields within the volume are obtained from Maxwell's coupled firstorder equations. The electric field is given by

$$
\frac{\partial(\varepsilon \boldsymbol{E}(\mathbf{r}, t))}{\partial t}=\nabla \times \mu^{-1} \boldsymbol{B}(\mathbf{r}, t)-\boldsymbol{J}(\mathbf{r}, t), \quad \mathbf{r} \in V
$$

and the magnetic flux is given by

$$
\frac{\partial(\boldsymbol{B}(\mathbf{r}, t))}{\partial t}=-\nabla \times \boldsymbol{E}(\mathbf{r}, t), \quad \mathbf{r} \in V .
$$

The current $\boldsymbol{J}(\mathbf{r}, t)$ is any impressed electric or magnetic currents within the volume. This term can be used to create a scattered-field formulation by setting it equal to

$$
\boldsymbol{J}(\mathbf{r}, t)=\frac{\partial\left(\left(\varepsilon-\varepsilon_{0}\right) \boldsymbol{E}_{\text {inc }}(\mathbf{r}, t)\right)}{\partial t}, \quad \mathbf{r} \in V .
$$

where $\varepsilon$ is the permittivity of a dielectric region, $\varepsilon_{0}$ is the permittivity of free space, and $\boldsymbol{E}_{\text {inc }}(\mathbf{r}, t)$ is the incident electric field.

To generate a system of equations, Ampere's law (1) is tested with some testing function $\boldsymbol{w}$. After integration-byparts, (1) becomes

$$
\begin{aligned}
\int_{V} \frac{\partial(\varepsilon \boldsymbol{E}(\mathbf{r}, t))}{\partial t} \cdot \boldsymbol{w}(\mathbf{r}) d V & =\int_{S} \mu^{-1} \boldsymbol{w} \cdot(\boldsymbol{B}(\mathbf{r}, t) \times \hat{\boldsymbol{n}}) d S \\
& -\int_{V} \boldsymbol{J}(\mathbf{r}, t) \cdot \boldsymbol{w}(\mathbf{r}) d V .
\end{aligned}
$$

For open region problems, the surface integral term in (4) can be used to truncate the finite element mesh with the firstorder absorbing boundary condition (ABC). After applying vector identities and approximating the relationship between the magnetic field and electric field with the Sommerfeld radiation condition, the first-order $\mathrm{ABC}$ is given as

$$
\begin{gathered}
\int_{S} \mu^{-1} \boldsymbol{w} \cdot(\boldsymbol{B} \times \hat{\boldsymbol{n}}) d S=\int_{S} \mu^{-1} \boldsymbol{w} \cdot \int_{t} \nabla \times \boldsymbol{E} \times \hat{\boldsymbol{n}} d t d S \\
\rightarrow-\frac{1}{Z} \int_{S}(\hat{\boldsymbol{n}} \times \boldsymbol{w}) \cdot(\hat{\boldsymbol{n}} \times \boldsymbol{E}) d S,
\end{gathered}
$$

where $\mathrm{Z}$ is the intrinsic impedance of free space.

Instead of the first-order ABC, a more accurate method of truncating the finite element mesh can be used. The interior $\mathbf{E}$ and $\mathbf{B}$ fields are used to compute equivalent surface magnetic and electric currents $\mathbf{J}$ and $\mathbf{M}$ on an inner source surface $\mathrm{S}_{\mathrm{s}}$, slightly inside the outer, radiative boundary of the mesh,

$$
\boldsymbol{M}=-\boldsymbol{E} \times \hat{\boldsymbol{n}} \text { and } \boldsymbol{J}=\hat{\boldsymbol{n}} \times \boldsymbol{H} \quad \text { for } \boldsymbol{r} \in S_{s}
$$

These equivalent currents are then used to find either the electric field $\boldsymbol{E}(\mathbf{r}, t)$ or the magnetic field $\boldsymbol{H}(\mathbf{r}, t)$ on the outer boundary of the mesh by first representing the fields in terms of the mixed- potentials,

$$
\begin{aligned}
\mathbf{E}^{s}(\mathbf{r}, t) & =-\frac{\partial}{\partial t} \mathbf{A}(\mathbf{r}, t)-\nabla \Phi(\mathbf{r}, t)-\frac{1}{\varepsilon} \nabla \times \boldsymbol{F}(\mathbf{r}, t) \\
\boldsymbol{H}^{s}(\mathbf{r}, t) & =-\frac{\partial}{\partial t} \boldsymbol{F}(\mathbf{r}, t)-\nabla \Psi(\mathbf{r}, t)+\frac{1}{\mu} \nabla \times \mathbf{A}(\mathbf{r}, t)
\end{aligned}
$$

and then expressing the time-delayed potentials in terms of the equivalent currents

$$
\begin{gathered}
\frac{\partial \mathbf{A}(\mathbf{r}, t)}{\partial t}=\frac{\mu}{4 \pi} \int_{S} \frac{\dot{\boldsymbol{J}}\left(\mathbf{r}^{\prime}, \tau\right)}{R} d S^{\prime}, \\
\Phi(\mathbf{r}, t)=-\frac{1}{4 \pi \varepsilon} \int_{S}^{\tau} \int_{0}^{\tau} \nabla_{S}^{\prime} \cdot \boldsymbol{J}\left(\mathbf{r}^{\prime}, t^{\prime}\right) d t^{\prime} d S^{\prime}, \\
\frac{\partial \boldsymbol{F}(\mathbf{r}, t)}{\partial t}=\frac{\varepsilon}{4 \pi} \int_{S} \frac{\dot{\boldsymbol{M}}\left(\mathbf{r}^{\prime}, \tau\right)}{R} d S^{\prime},
\end{gathered}
$$

and

$$
\Psi(\mathbf{r}, t)=-\frac{1}{4 \pi \mu} \int_{S}^{\tau} \int_{0}^{\tau} \nabla_{S}^{\prime} \cdot \boldsymbol{M}\left(\mathbf{r}^{\prime}, t^{\prime}\right) d t^{\prime} d S^{\prime} .
$$

In (9)-(12), $R=\left|\mathbf{r}-\mathbf{r}^{\prime}\right|$ is the distance between a source and observation location and $\tau=t-\left|\boldsymbol{r}-\mathbf{r}^{\prime}\right| / c$ is the time delay between points located on the source surface and observation surface. In addition, the quantities $\mu$ and $\varepsilon$ are the permeability and permittivity, respectively, of the medium between the two layers which must be the same as the region outside the outer boundary. The variable $c$ is the speed of light in this region. 
Each of the different radiation boundary conditions can be applied by evaluating either the electric, magnetic or unified field at the outer surface using the sources from the inner surface. For instance, a Dirichlet radiating boundary condition is applied by evaluating the tangential electric field on the outer surface, similar to the formulation in [3] for FDTD,

$$
-\hat{\mathbf{n}} \times \hat{\boldsymbol{n}} \times\left.\boldsymbol{E}\right|_{S}=\boldsymbol{E}_{\text {tan }}(\boldsymbol{J}, \boldsymbol{M}) .
$$

Substituting (9)-(11) into (7) and evaluating the electric field using (13), the Dirichlet boundary condition is given by

$$
\begin{aligned}
-\hat{\mathbf{n}} \times \hat{\boldsymbol{n}} \times\left.\boldsymbol{E}(\boldsymbol{r}, t)\right|_{S}= & \frac{\mu}{4 \pi} \int_{S_{s}^{\prime}} \frac{\dot{\boldsymbol{J}}\left(\mathbf{r}^{\prime}, \tau\right)}{R} d S^{\prime}-\frac{\nabla}{4 \pi \varepsilon} \int_{S_{s}^{\prime}} \int_{0}^{\tau} \frac{\nabla_{S}^{\prime} \cdot \boldsymbol{J}\left(\mathbf{r}^{\prime}, t^{\prime}\right)}{R} d t^{\prime} \\
& +\frac{1}{4 \pi} \int_{S_{s}^{\prime}} \frac{\nabla \times \boldsymbol{M}\left(\mathbf{r}^{\prime}, \tau\right)}{R} d S_{s}^{\prime}
\end{aligned}
$$

Likewise, the Neumann's boundary condition can be applied by evaluating the magnetic field $\boldsymbol{H}(\mathbf{r}, t)$. By recognizing the surface integral term in (4) can be written in term of the magnetic field

$$
-\int_{S} \mu^{-1} \boldsymbol{w} \cdot(\boldsymbol{B} \times \hat{\boldsymbol{n}}) d S=-\int_{S} \boldsymbol{n} \times \boldsymbol{w} \cdot \mathbf{H} d S,
$$

which is found from (7)-(10) as

$$
\begin{gathered}
-\boldsymbol{H}_{\text {tan }}^{s}(\boldsymbol{r}, t)=\frac{\varepsilon}{4 \pi} \int_{S_{\mathrm{s}}^{\prime}} \frac{\dot{\boldsymbol{M}}\left(\mathbf{r}^{\prime}, \tau\right)}{R} d S^{\prime}-\frac{\nabla}{4 \pi \mu} \int_{S_{s}^{\prime}} \int_{0}^{\tau} \frac{\nabla_{s}^{\prime} \cdot \boldsymbol{M}\left(\mathbf{r}^{\prime}, t^{\prime}\right)}{R} d t^{\prime} d S_{s}^{\prime} \\
-\frac{1}{4 \pi} \int_{S_{s}^{\prime}} \frac{\nabla \times \boldsymbol{J}\left(\mathbf{r}^{\prime}, \tau\right)}{R} d S_{s}^{\prime}
\end{gathered}
$$

Alternately, the proposed unified boundary condition (UBC) can be derived by combining the Dirchlet's and Neumann's boundary conditions together. Effectively, the electric and magnetic fields are superimposed into a U-field as

$$
\boldsymbol{U}(\boldsymbol{r}, t)=-\frac{1}{c} \hat{\boldsymbol{n}} \times \hat{\boldsymbol{n}} \times \boldsymbol{E}(\boldsymbol{r}, t)+\hat{\boldsymbol{n}} \times \boldsymbol{H}(\boldsymbol{r}, t),
$$

where a weighted sum of the tangential magnetic and electric fields from the finite-elements will be forced to match the same weighted sum computed from the boundary elements.

Substituting (17) into the surface integral term on the right hand side of (4), the resulting unified boundary condition becomes

$$
-\int_{S} \mu^{-1} \boldsymbol{w} \cdot(\boldsymbol{B} \times \hat{\boldsymbol{n}})=-\frac{1}{Z} \int_{S}(\hat{\mathbf{n}} \times \boldsymbol{w}) \cdot(\hat{\mathbf{n}} \times \boldsymbol{E})+\int_{S} \mu^{-1} \boldsymbol{w} \cdot \boldsymbol{U} .
$$

Note, that the formulation contains the first-order $A B C$ if the $\boldsymbol{U}$ field is zero and $\boldsymbol{B}$ and $\boldsymbol{E}$ are the magnetic and electric fields produced by the finite-elements at the outer surface. The complete $\boldsymbol{U}$ field expression in terms of fields produced by the equivelent sources is then substituted into the surface integral right hand side of (18) as

$$
\int_{S} \mu^{-1} \boldsymbol{w} \cdot(\boldsymbol{U}) d S=\frac{1}{C} \int_{S} \boldsymbol{w} \cdot\left(\boldsymbol{E}_{\text {tan }}\right) d S-\int_{S} \hat{\mathbf{n}} \times \boldsymbol{w} \cdot(\boldsymbol{H}) d S .
$$

Examining (19), the first boundary element term is the same as that filled for the Dirichlet boundary condition times a scaling factor, while the second is that used for the Neumann boundary condition.

\section{NUMERICAL IMPLEMENTATION}

In this section, a numerical implementation of the twosurface hybrid is detailed. The volume is meshed with vector finite elements and solved using an explicit, time-domain finite element approach. The interior and exterior surfaces are meshed with surface elements upon which equivalent currents

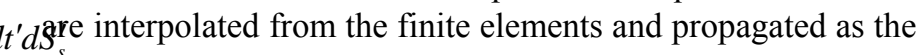
U-field to the outer surface using the time-domain method of moments.

\section{A. Vector Finite Element Time Domain}

The vector finite element time-domain method is used with a Galerkin procedure to convert the weak form of Maxwell's equations to a semidiscrete coupled system of ordinary differential equations using vector finite elements. The VFETD method uses curl-conforming vector "edge" finite elements that have tangential continuity across elements as a basis for the electric field

$$
\boldsymbol{E}=\sum_{j=1}^{N_{E}} e_{j} \boldsymbol{W}_{j}(\mathbf{r})
$$

and vector "face" finite elements with normal continuity across elements as a basis for the magnetic flux density

$$
\boldsymbol{B}=\sum_{j=1}^{N_{B}} b_{j} \boldsymbol{F}_{j}(\mathbf{r}) \text {. }
$$

The properties of these vector finite elements are discussed in more detail in [8]. By testing (1) with a curl conforming function $\mathbf{W}$ and substituting the expansions for the $\mathbf{E}$ and $\mathbf{B}$ fields (20) and (21) into (1), a weak formulation of Ampere's law is obtained,

$$
\frac{\partial}{\partial t}\left(\boldsymbol{W}_{i}, \varepsilon \boldsymbol{E}\right)=\left(\nabla \times \boldsymbol{W}_{i}, \mu^{-1} \boldsymbol{B}\right)-\left(\boldsymbol{W}_{i}, \boldsymbol{J}\right), \quad i=1,2, \ldots, N_{E}
$$

and

$$
\frac{\partial}{\partial t}\left(\boldsymbol{F}_{i}, \mu^{-1} \boldsymbol{B}\right)=-\left(\boldsymbol{F}_{i}, \nabla \times \boldsymbol{E}\right), \quad i=1,2, \ldots, N_{F}
$$

where $\mathrm{N}_{\mathrm{E}}$ and $\mathrm{N}_{\mathrm{F}}$ are the number of internal edges and faces, respectively. This leads to the system of ordinary differential equations,

$$
C \frac{\partial \boldsymbol{e}}{\partial t}=K^{T} \boldsymbol{b}-Q \boldsymbol{j}
$$

and

$$
G \frac{\partial \boldsymbol{b}}{\partial t}=-T \boldsymbol{e}
$$

where $\boldsymbol{b}=\left[b_{1}, b_{2}, \ldots, b_{\mathrm{NF}}\right]^{\mathrm{T}}, \boldsymbol{e}=\left[e_{1}, e_{2}, \ldots, e_{\mathrm{NE}}\right]^{\mathrm{T}}$ and the matrices are given by 


$$
\begin{gathered}
C_{i j}=\left(\varepsilon \boldsymbol{W}_{i}, \boldsymbol{W}_{j}\right), \quad G_{i j}=\left(\mu^{-1} \boldsymbol{F}_{i}, \boldsymbol{F}_{j}\right), \\
K_{i j}=\left(\mu^{-1} \nabla \times \boldsymbol{W}_{i}, \boldsymbol{F}_{j}\right) \text { and } Q_{i j}=\left(\boldsymbol{W}_{i}, \boldsymbol{W}_{j}\right), \\
T_{i j}=\left(\boldsymbol{F}_{j}, \nabla \times \boldsymbol{W}_{i}\right) .
\end{gathered}
$$

A symplectic time integration scheme similar to [1] is then used to discretize the equations in time. The time derivatives are approximated using central difference. The electric fields are calculated at whole time steps and the magnetic fields calculated at half-time steps as

$$
(C) \boldsymbol{e}^{n+1}=\left(\Delta t K^{T}\right) \boldsymbol{b}^{n+1 / 2}-(\Delta t Q) \boldsymbol{j}^{n+1}+(C) \boldsymbol{e}^{n}
$$

and

$$
(G) \boldsymbol{b}^{n+3 / 2}=-\left(\Delta t T^{T}\right) \boldsymbol{e}^{n}+(G) \boldsymbol{b}^{n+1 / 2} .
$$

While these electric and magnetic fields are being updated, the radiation boundary condition must be applied. For the electric field boundary case, it is applied as a Dirichlet boundary condition on the solution of (27). For the magnetic field boundary or unified boundary condition, it is added to the right hand side of (27).

At every iteration, the fields must be used to generate the equivalent surface currents as shown in Fig. 2. For the magnetic current, this is done by directly using the degrees of freedom from the electric field curl-conforming bases as the degrees of freedom for the magnetic current surface divergence conforming basis functions. For the electric current, it is done through spatial and temporal interpolation.

\section{B. Time Domain Method of Moments}

The time domain method of moments is used to solve the integral equations for the electric and magnetic fields used in the unified boundary condition. A series of time-delayed matrices for the propagation of fields from the inner source surface to the outer, radiative surface is constructed and applied using a sub-cycle method versus the explicit time step used in the VFEMTD.

A numerical solution to (14), (16) or (19) is obtained by discretizing the surfaces with surface elements and expanding both the unknown current distribution for $\mathbf{J}$ and $\mathbf{M}$ across each element in a set of basis functions in time and space as

$$
\boldsymbol{J}(\mathbf{r}, t)=\sum_{n=1}^{N} \sum_{j=1}^{T} J_{n}^{(j)} \boldsymbol{\Lambda}_{n}(\mathbf{r}) T_{j}(t)
$$

and

$$
\boldsymbol{M}(\mathbf{r}, t)=\sum_{n=1}^{N} \sum_{j=1}^{T} M_{n}^{(j)} \boldsymbol{\Lambda}_{n}(\mathbf{r}) T_{j}(t),
$$

where $J_{n}^{(j)}$ and $M_{n}^{(j)}$ is the $n$th unknown current coefficients at time $t_{j}$. $\mathrm{N}$ is the number of edges on the inner surface and $T$ is the number of time steps. The temporal basis function can be any higher-order interpolatory function but a piece-wise linear function has been used.

In order to preserve the functional spaces occupied by the electric and magnetic fields, a complimentary surface space is defined for the equivalent electric and magnetic surface currents. The spatial component of the current distribution is thus modeled with a spatial basis function $\boldsymbol{\Lambda}_{\mathrm{n}}(\boldsymbol{r})$ based on the same discrete space as used in the 1-forms for the finite elements. The current across a boundary element is modeled with a twisted 1-form basis functions ( $\mathbf{\Lambda}$ ), which are divergence-conforming edge basis functions. They are derived from the higher-order interpolatory 1-form basis functions and are "twisted" in the sense that they are rotated $90^{\circ}$ from the 1-form basis function. The 1-form basis functions $(\boldsymbol{w})$ are derived from the same curl conforming polynomial spaces as the 1 -form volume basis functions. The discrete surface twisted 1-forms $(\boldsymbol{\Lambda})$ is derived from the 1form basis function $(w)$ as

$$
\Lambda=w \times n
$$

These bases accurately represent surface divergence, and differ from the RWG basis function [9] only by a scale factor.

Substituting (29) and (30) into $(9,10,11,12)$ and rewriting the electric and magnetic potentials in a generalized manner $(\boldsymbol{B}, B$, $\Delta \times \boldsymbol{B})$ allows the generalized potential forms to be written in terms of sums of partial potentials as

$$
\begin{array}{r}
\frac{\partial \boldsymbol{B}(\mathbf{r}, t)}{\partial t}=\sum_{n=1}^{N} \sum_{j=j_{k}^{\text {min }}}^{j_{k}^{\text {max }}} I_{n}^{(j)} \int_{S} \frac{\boldsymbol{\Lambda}_{n}\left(\mathbf{r}^{\prime}\right)}{4 \pi R} \dot{T}_{j}(\tau) d S^{\prime}, \\
B(\mathbf{r}, t)=-\sum_{n=1}^{N} \sum_{j=j_{k}^{\text {min }}}^{j_{j}^{\text {max }}} I_{n}^{(j)} \int_{S} \frac{\nabla_{S}^{\prime} \cdot \boldsymbol{\Lambda}_{n}\left(\mathbf{r}^{\prime}\right)}{4 \pi R} \int_{0}^{\tau} T_{j}\left(t^{\prime}\right) d t^{\prime} d S^{\prime}
\end{array}
$$

and

$$
\nabla \times \boldsymbol{B}(\mathbf{r}, t)=-\sum_{n=1}^{N} \sum_{j=j_{k}^{\text {min }}}^{j_{k}^{\text {max }}} I_{n}^{(j)} \int_{S} \nabla G\left(\boldsymbol{r}, \boldsymbol{r}^{\prime}, t\right) \cdot \boldsymbol{\Lambda}_{n}\left(\mathbf{r}^{\prime}\right) T_{j}\left(\tau^{\prime}\right) d S^{\prime}
$$

where

$$
\nabla G\left(\boldsymbol{r}, \boldsymbol{r}^{\prime}, t\right)=\left(\frac{1}{4 \pi c} \frac{\partial}{\partial t} \frac{\hat{R}}{R}+\frac{\hat{R}}{4 \pi R^{2}}\right) \times \overline{\boldsymbol{I}} .
$$

Notice that the summation limits in (32)-(34) over the temporal basis have been replaced by a finite interval $\left[j_{k}{ }^{\mathrm{min}}, j_{k}{ }^{\mathrm{max}}\right]$, which stands for the delay range in terms of a time interval for a given element-element pair found between the two surfaces.

A suitable testing scheme must be adopted to solve the UBC in (19) using the method of moments. In this formulation, standard Galerkin testing functions are used in space, evaluated on the outer surface via a higher-order Gaussian rule. Note the different testing functions, surface basis function $w$ and $\Lambda$, are used to evaluate the electric and magnetic field integral equations, respectively. Also, point matching in time is applied but done using a subcycled time step $\Delta t_{i}{ }^{\prime}$ that can operate at multiples values of the explicit, finite element time step. Applying this testing procedure to (19) leads to the tested $\mathbf{U}$ field equation

$$
\left(\boldsymbol{w}_{m}, \mu^{-1} \boldsymbol{U}\left(t_{i}^{\prime}\right)\right)=\left(\boldsymbol{w}_{m}, c^{-1} \boldsymbol{E}_{\tan }\left(t_{i}^{\prime}\right)\right)+\left(\Lambda_{m}, \boldsymbol{H}\left(t_{i}^{\prime}\right)\right)
$$

where $\mathrm{m}=1, \ldots \mathrm{Ns}$, and Ns is the number of edge unknowns on the outer surface. After substituting the generalized partial 
potentials (32)-(34) into (35), a linear system of time-delayed matrices is obtained for the $\mathbf{U}$ field which is updated at each boundary element time step $\Delta \mathrm{t}^{\prime}$ as

$$
U_{m}^{\left(i^{\prime}\right)}=\sum_{j^{\prime}=0}^{T} \sum_{m=1}^{N} \sum_{n=1}^{N}\left[\begin{array}{l:l}
Z_{E H\left(\boldsymbol{J}_{n}\right)}^{\left(j^{\prime}\right)} & Z_{\boldsymbol{E}\left(\boldsymbol{M}_{n}\right)}^{\left(j^{\prime}\right)}
\end{array}\right]\left[\begin{array}{c}
J_{n}^{\left(i^{\prime}-j^{\prime}\right)} \\
\hdashline M_{n}^{\left(i^{\prime}-j^{\prime}\right)}
\end{array}\right] .
$$

Thus a correction to the first-order $\mathrm{ABC}$ is obtained from (36), where the U-field $U_{m}{ }^{\left({ }^{\prime}\right)}$ is updated at each time step $t_{i}{ }^{\prime}$ due to the time-delayed electric and magnetic currents $\left[J_{n}^{\left(i^{\prime}-j^{\prime}\right)}\right.$ $\left.M_{n}{ }^{\left(i^{\prime}-j^{\prime}\right)}\right]$ from previous time steps $t_{i}{ }^{\prime}=t_{j}{ }^{\prime}-R / c$. The other radiation boundary conditions can also be employed using similar field expressions. The time-delayed system submatrices $\boldsymbol{Z}_{m, n}{ }^{\left({ }^{\prime}\right)}$ in (36), are given by

$$
\begin{aligned}
& {\left[Z_{E \boldsymbol{E}\left(\boldsymbol{J}_{n}\right)}^{\left(j^{\prime}\right)}: Z_{\boldsymbol{E H}\left(\boldsymbol{M}_{n}\right)}^{\left(j^{\prime}\right)}\right]=} \\
& {\left[c^{-1} Z_{\boldsymbol{w}_{m}, \boldsymbol{E}\left(\boldsymbol{J}_{n}\right)}^{\left(\boldsymbol{j}^{\prime}\right)}+Z_{\boldsymbol{\Lambda}_{m}, \boldsymbol{H}\left(\boldsymbol{J}_{n}\right)}^{\left(j^{\prime}\right)}: c^{-1} Z_{\boldsymbol{w}_{m}, \boldsymbol{E}\left(\boldsymbol{M}_{n}\right)}^{\left(j^{\prime}\right)}+Z_{\boldsymbol{\Lambda}_{m}, \boldsymbol{H}\left(\boldsymbol{M}_{n}\right)}^{\left(j^{\prime}\right)}\right]}
\end{aligned}
$$

where

$$
\begin{aligned}
Z_{\boldsymbol{w}_{m}, E\left(J_{n}\right)}^{j^{\prime}}= & \iint_{S}\left[\frac{\mu}{4 \pi} \frac{\boldsymbol{w}_{m}(\mathbf{r}) \cdot \boldsymbol{\Lambda}_{n}\left(\mathbf{r}^{\prime}\right)}{R} \dot{T}_{j^{\prime}}\left(\tau^{\prime}\right)\right. \\
& \left.+\frac{1}{4 \pi \varepsilon} \frac{\boldsymbol{w}_{m}(\mathbf{r}) \cdot \nabla_{S} \nabla_{S}^{\prime} \cdot \boldsymbol{\Lambda}_{n}\left(\mathbf{r}^{\prime}\right)}{R} \int_{0}^{\tau^{\prime}} T_{j^{\prime}}\left(t^{\prime}\right) d t^{\prime}\right] d S^{\prime} d S
\end{aligned}
$$

$$
\begin{gathered}
Z_{\boldsymbol{w}_{m}, \boldsymbol{E}\left(\boldsymbol{M}_{n}\right)}^{j^{\prime}}=-\int_{S} \int_{S^{\prime}}\left[\boldsymbol{w}_{m}(\mathbf{r}) \cdot \nabla G\left(\boldsymbol{r}, \mathbf{r}^{\prime}, t\right) \cdot \boldsymbol{\Lambda}_{n}\left(\mathbf{r}^{\prime}\right) T_{j^{\prime}}\left(\tau^{\prime}\right)\right] d S^{\prime} d S, \\
Z_{\boldsymbol{\Lambda}_{m}, \boldsymbol{H}\left(\boldsymbol{J}_{n}\right)}^{j^{\prime}}=\int_{S} \int\left[\boldsymbol{\Lambda}_{S^{\prime}}\left[\boldsymbol{\Lambda}_{m}(\mathbf{r}) \cdot \nabla G\left(\boldsymbol{r}, \mathbf{r}^{\prime}, t\right) \cdot \boldsymbol{\Lambda}_{n}\left(\mathbf{r}^{\prime}\right) T_{j^{\prime}}\left(\tau^{\prime}\right)\right] d S^{\prime} d S,\right.
\end{gathered}
$$

and

$$
\begin{aligned}
Z_{\boldsymbol{\Lambda}_{m}, \boldsymbol{H}\left(\boldsymbol{M}_{n}\right)}^{j^{\prime}}= & \int_{S} \int_{S^{\prime}}\left[\frac{\varepsilon}{4 \pi} \frac{\boldsymbol{\Lambda}_{m}(\mathbf{r}) \cdot \boldsymbol{\Lambda}_{n}\left(\mathbf{r}^{\prime}\right)}{R} \dot{T}_{j^{\prime}}\left(\tau^{\prime}\right)\right. \\
& \left.+\frac{1}{4 \pi \mu} \frac{\nabla_{S} \cdot \boldsymbol{\Lambda}_{m}(\mathbf{r}) \nabla_{S}^{\prime} \cdot \boldsymbol{\Lambda}_{n}\left(\mathbf{r}^{\prime}\right)}{R} \int_{0}^{\tau^{\prime}} T_{j^{\prime}}\left(t^{\prime}\right) d t^{\prime}\right] d S^{\prime} d S
\end{aligned}
$$

The last final comment concerns the time step used for the integral equations. As mentioned previously, it need not be the same time step as the finite element time step. In particular, it can be sub-cycled at some larger multiple $\Delta t_{i}{ }^{\prime}$ of the finite element time step. This offers two advantages. The first is that this sub-sampling leads to faster time-stepping, because the matrix-vector products used to compute the electric and magnetic fields on the outer boundary do not need to be performed every time step. The second advantage is that the representation of $\mathrm{E}$ and $\mathrm{H}$ on the boundary can actually become more accurate, leading to a more stable solution. Because the time step needed for the finite element is often small, such that light takes several timesteps to cross one face of an element, standard integration techniques have trouble accurately evaluating the potential integrals. For pure BEM problems, the timestep required for finite-element stability would lead to unstable results. By using a multiple of the finite element time step for the BEM timestep, the time integration testing in time becomes more accurate, removing late-time instabilities. This same sub-cycling method used in [5] for FDTD to reduce complexity, is also shown here to improves stability and overall accuracy of the technique.

\section{ApPliCATIONS AND RESUltS}

Several 3D hybrid meshes are simulated to validate the proposed unified boundary condition presented in this paper. In particular, the results will demonstrate the accuracy of the solution over the first-order $\mathrm{ABC}$. More importantly, the UBC will show long-term stability of the solution over the previously derived radiation boundary conditions. All cases considered here suggest improved late-time stability over the Dirchlet's and Neumann's boundary conditions. Two different types of excitation problems are considered, an internal source problem and a scattering problem. All simulations were performed on a LINUX based cluster computer.

To validate our new hybrid boundary element - finite element code, we have chosen to first solve a problem which has a known analytic solution. The problem chosen is the same as that used in [3] for verification of an FDTD-BEM hybrid. An electric dipole oriented along the $\mathrm{z}$-axis is assumed to be located at the origin. Its dipole moment is taken to be

$$
\boldsymbol{p}(\boldsymbol{r}, t)=f(t) \delta(\boldsymbol{r}) \hat{\mathbf{z}} .
$$

The resultant fields are

$$
\begin{gathered}
\boldsymbol{E}(\boldsymbol{r}, t)=\frac{\{3(\boldsymbol{r} \cdot \mathbf{z}) \boldsymbol{r}-\mathbf{z}\}}{4 \pi r^{3}}\left[f+\tau \partial_{t} f\right]+\frac{\{\boldsymbol{r} \times(\boldsymbol{r} \times \hat{\mathbf{z}})\}}{4 \pi \varepsilon_{0} c^{2} r}\left[\partial_{t}^{2} f\right], \\
\boldsymbol{H}(r, t)=-\frac{(\boldsymbol{r} \times \hat{\mathbf{z}})}{4 \pi r^{2}}\left[\partial_{t} f+\tau \partial_{t}^{2} f\right],
\end{gathered}
$$

where $\boldsymbol{r}=(x, y, z)$ is our position in space, $\mathrm{c} \tau=|\boldsymbol{r}|=\mathrm{r}$ and [f] $=\mathrm{f}(\mathrm{t}-\tau)$ denotes evaluation at the retarded time $\mathrm{t}-\tau$.

The driving function $\mathrm{f}$ is designed to turn on and then turn off over a period of $50 \mathrm{~ns}$. The response is a pulse that radiates outward through space. This function is defined by $\mathrm{f}(\mathrm{t})=$ $\mathrm{g}(\beta \mathrm{t})$, where $\beta=2.0 \times 10^{7} \mathrm{sec}^{-1}$, and where

$$
\begin{aligned}
g(x) & =0, & & x<0, x>1, \\
& =32 x^{3}-48 x^{4}, & & 0 \leq x \leq 1 / 2, \\
& =32(1-x)^{3}-48(1-x)^{4}, & & 1 / 2 \leq x \leq 1 .
\end{aligned}
$$

We observe that this function is continuous and has continuous first and second derivatives, but discontinuous third derivatives. Figures 2 and 3 show the time history of the electric and magnetic field solutions for this dipole pulse at the test point location $(0.91573,0.27778,0.29028)$, which was chosen to avoid any symmetry planes of the solution. 


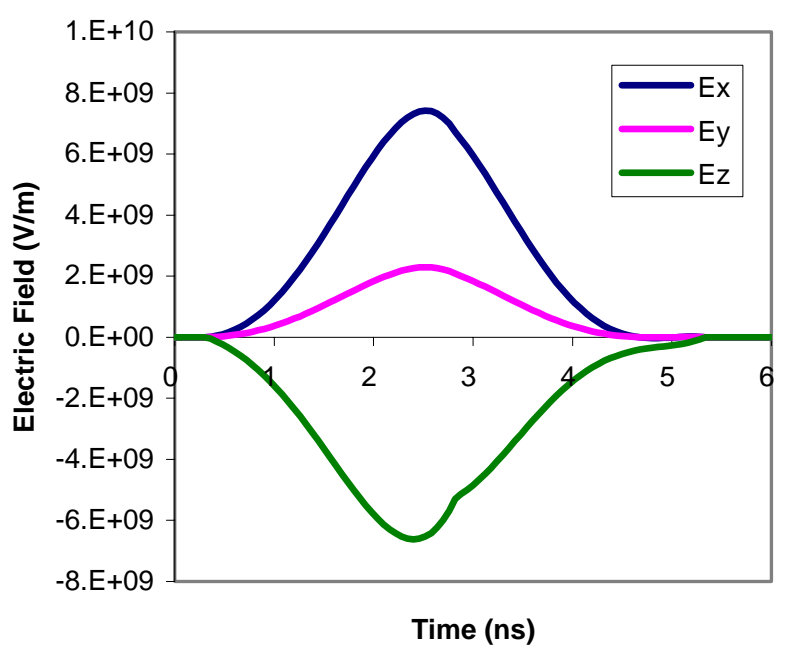

Figure 2. Exact solution for the electric field from the pulsed dipole.

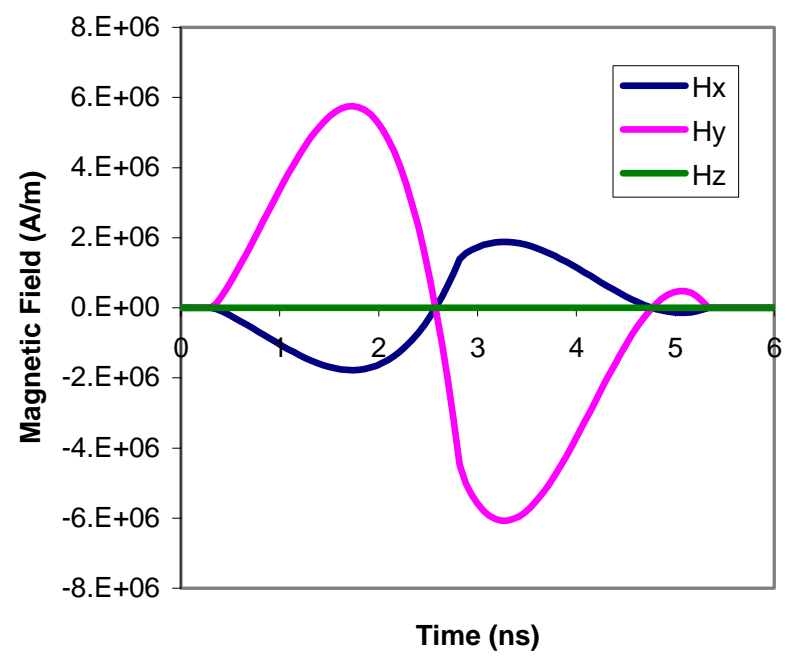

Figure 3: Exact solution for the magnetic field from the pulsed dipole.

This problem is solved over the volume between two concentric spheres with radii of 0.25 and 1.0, respectively. Figure 4 shows a typical hexahedral mesh for this problem. The problem is excited by specifying the exact field solution on the interior spherical surface. At the outer surface (radius = 1.0 ) the boundary element hybrid boundary condition is used to truncate the mesh. This outer surface is relatively close to the dipole source. This will cause difficulties with conventional radiation boundary conditions such as "far-field" impedance conditions. A sequence of refined meshes is used to investigate accuracy, convergence and stability. Table 1 shows the number of nodes and elements in each of the different meshes.
Table1. Number of nodes and elements for different test meshes.

\begin{tabular}{|l|l|l|}
\hline $\begin{array}{l}\text { Mesh } \\
\text { ID }\end{array}$ & Nodes & Elements \\
\hline 16 & 686 & 576 \\
\hline 32 & 5018 & 4608 \\
\hline 64 & 38450 & 36864 \\
\hline 128 & 301154 & 294912 \\
\hline 256 & 2384066 & 2359296 \\
\hline
\end{tabular}

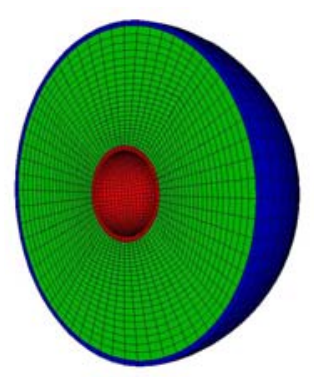

Figure 4. Half of the typical hexahedral mesh for the dipole64 problem.

The hybrid radiating boundary condition is compared to a conventional first-order accurate absorbing boundary condition $(\mathrm{ABC})$, placed at the outer surface. Figure 5 shows the relative error for the same Ez component for the hybrid method for the various meshes together with the relative error for the ABC method on the finest mesh. The hybrid method is relatively accurate even for the coarse meshes and is converging to the exact solution as the meshes are refined. We observe almost a 1000 -fold less error for the hybrid solution than for the ABC on the Sphere 128 mesh.

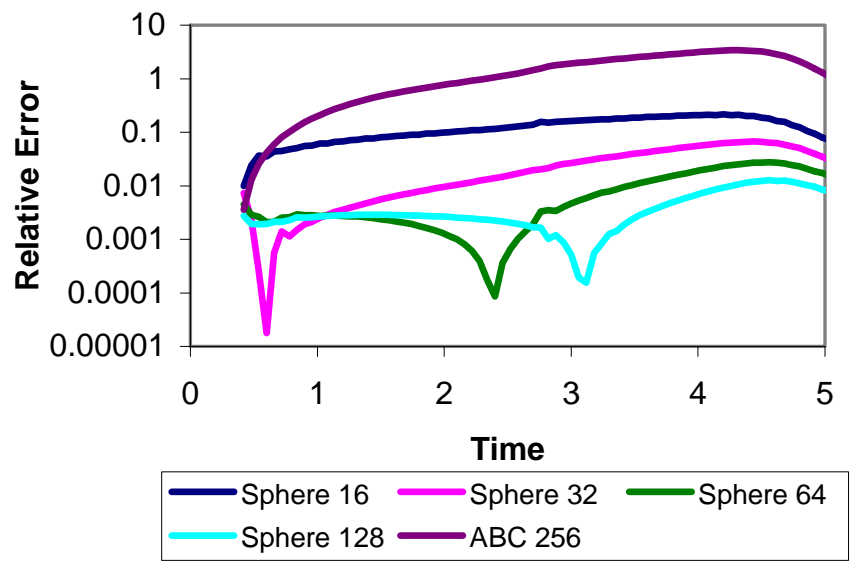

Figure 5. Relative Error for the hybrid method and conventional $\mathrm{ABC}$ boundary conditions. 
The formulation section presents three separate ways to use the boundary elements to truncate the finite-element mesh; a Dirichlet, Neumann, or combined approach. These three methods were tested on the 64-size sphere problem. The results for Ez sampled at a point are shown in Figure 6. Both the Dirichlet and Neumann applications have stability problems fairly early in the time marching. Even the combined UBC without sub-cycling eventually displays late time instability. Only the UBC with sub-cycling remains stable at the noise floor late in the simulation. Because of the potential for instability in the other formulations, the UBC with subcyling is used for the rest of the examples in this paper.

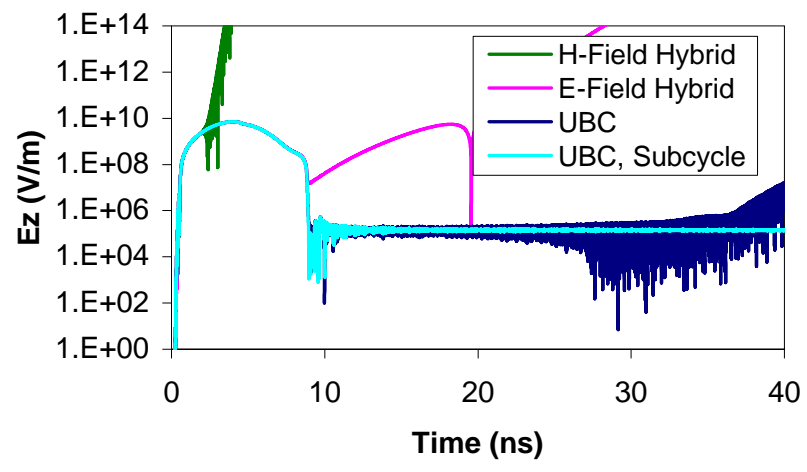

Figure 6. Stability comparison of the different methods for using the BEM to truncate the finite-elements.

There are two other effects that occur when sub-cycling is used: the solution accuracy changes, and the computational time is significantly affected. In general, when the boundary element computation is done less frequently, the accuracy degrades. However, the computational run-time decreases dramatically. So, in using sub-cycling, trade-offs must be made. Figure 7 shows how the maximum relative error for the Ez component degrades as the sub-cycling frequency changes. When the sub-cycling changes from 2 (every other finiteelement time step) to 16 , the relative maximum error increases by more than an order of magnitude. In contrast, Figure 8 shows how the computational efficiency increases. The computational time per time step decreases by a more than an order of magnitude.

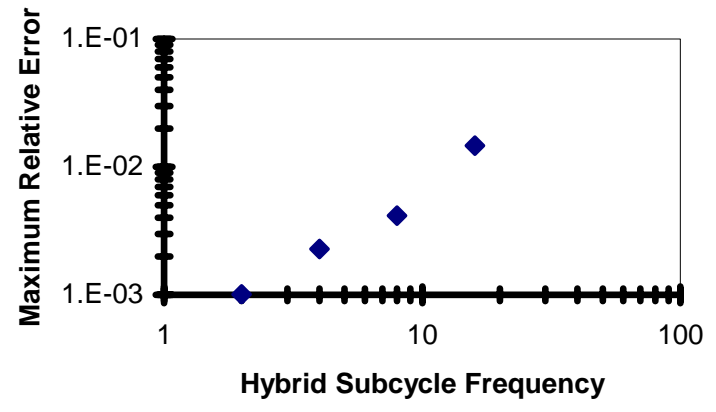

Figure 7. The maximum relative error increases with the subcycling frequency

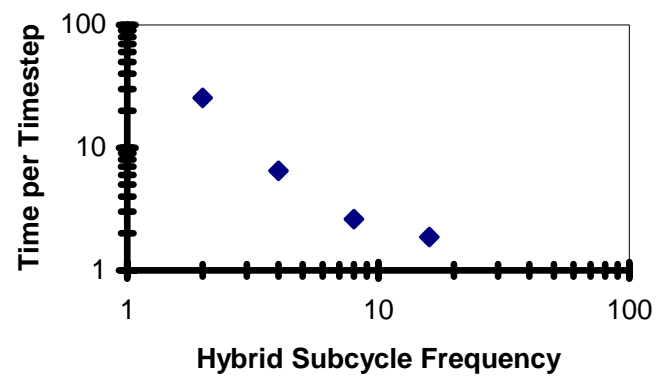

Figure 8 . The computational cost per time step decreases as the sbu-cycling frequency increases.

Figure 9 shows how the Ez component error magnitude behaves for the Sphere 32 problem as the sub-cycling frequency changes. In general, we have found that using a hybrid sub-cycling frequency of 4 or 8 does not degrade the accuracy unacceptably and dramatically improves the computational efficiency of the method.

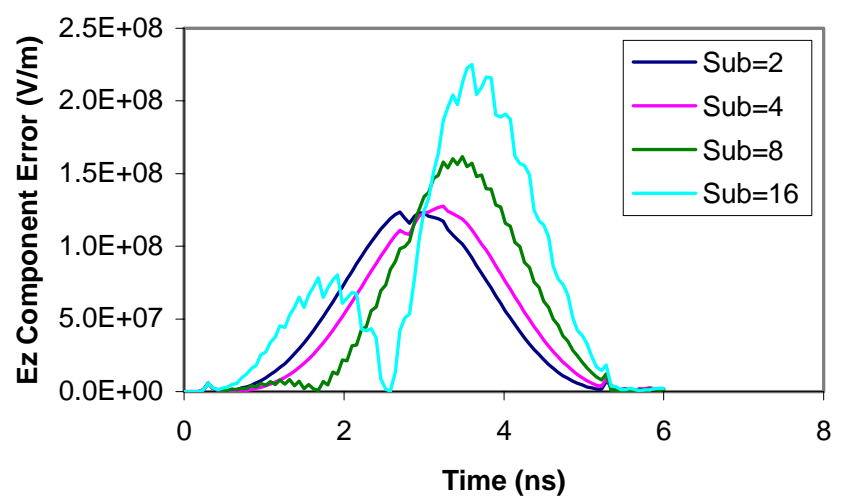

Figure 9. Ez component error behavior as the hybrid subcycling frequency changes.

The second test problem, scattering from a PEC sphere, was used to test the hybrid boundary condition for 
scattering problems. A hexahedral mesh including a $0.5 \mathrm{~m}$ radius spherical PEC surface, meshed with 96 quads, was used. For the hybrid case, the inner surface was placed one element away from the PEC sphere, and the outer surface one element from that. For the ABC case, the outer boundary was placed $4 \mathrm{~m}$ away from the sphere in order to obtain good results.

The sphere was hit with a plane wave polarized in the $\mathrm{x}$ direction incident from $-\mathrm{z}$. The plane wave was modulated by a Gaussian enveloped cosine, given by

$$
E_{x}(\boldsymbol{r})=\cos (\omega(t-\boldsymbol{k} \cdot \boldsymbol{r})) e^{\frac{-1\left(t-t_{0}-\boldsymbol{k} \cdot \boldsymbol{r}\right)^{2}}{\tau^{2}}}
$$

where $\omega=2 \pi 100 \times 10^{6}, \boldsymbol{k}=3.33564 \times 10^{-9} \hat{\boldsymbol{x}}$, and $\tau=1.91 \times 10^{-8}$. Because the air padding around the sphere was almost eliminated for the hybrid mesh, the hybrid simulation ran much quicker. The ABC simulation took 18.3 minutes to run on a single processor, whereas the hybrid simulation only took 2.1 minutes to run. The results for the $E_{\theta}$ scattered field are shown below in Figure 10. While both the $\mathrm{ABC}$ and hybrid simulations show good agreement with the MIE series solution, the hybrid agreement is slightly better.

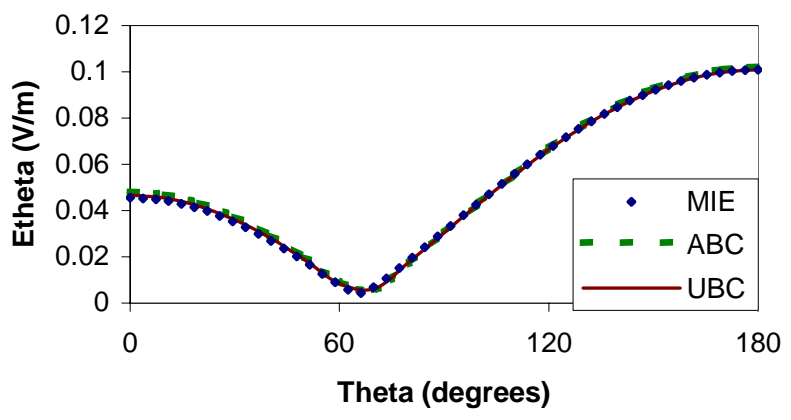

Figure 10. A comparison of the $E_{\theta}$ scattered fields from a PEC sphere using the MIE series, the ABC, and the hybrid formulation.

A final test case consisting of scattering from a rocket with a dielectric nosecone was simulated. The rocket, shown in Figure 11, consists of a $1 \mathrm{~m}$ long PEC cylinder of radius $0.4 \mathrm{~m}$ with a hemispherical dielectric nosecone with $\varepsilon_{r}=4$. In addition, four tapered fins were connected to the body. These fins taper from $0.35 \mathrm{~m}$ tall where they connect to the body to $0.25 \mathrm{~m}$ at $0.1 \mathrm{~m}$ away from the rocket body.

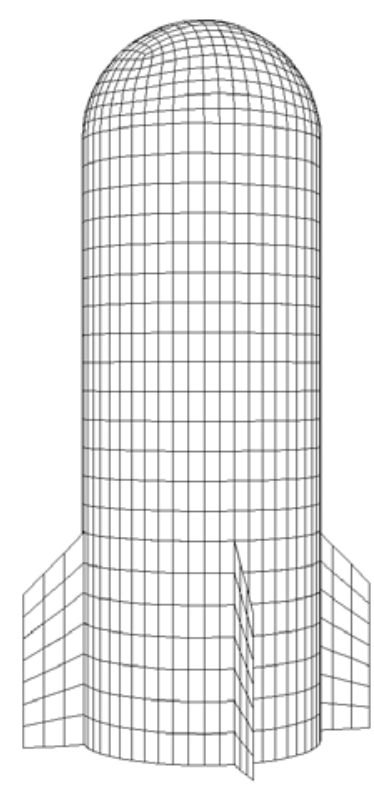

Figure 11. The rocket mesh used for the hybrid simulation

The rocket was hit with a Gaussian enveloped cosine plane wave incident from broadside polarized in $\mathrm{z}$, the long axis of the rocket. The center frequency of the plane wave was 350 $\mathrm{MHz}$, and the width factor $\tau=1.736 \times 10^{-9}$ was used. The rocket was meshed using a conformal hexahedral mesh, with the inner surface for the hybrid problem placed one cell away from the rocket, and the outer surface placed one cell away from the inner surface. For the ABC simulation, the rocket was meshed using a tetrahedral mesh, padded with air to 6 meters from the rocket. The hybrid mesh contained 24 thousand hexehedral elements, and the $\mathrm{ABC}$ mesh contained 5.5 million tetrahedra. A timestep of $2 \times 10^{-11} \mathrm{~s}$ was used for both methods, and the simulations were allowed to run 1600 timesteps. The hybrid problem took 45 minutes on 16 processors, while the ABC problem took 1 hour and 48 minutes on 64 processors. Both methods show fairly good agreement for the far field scattering, as shown in Figure 12. 


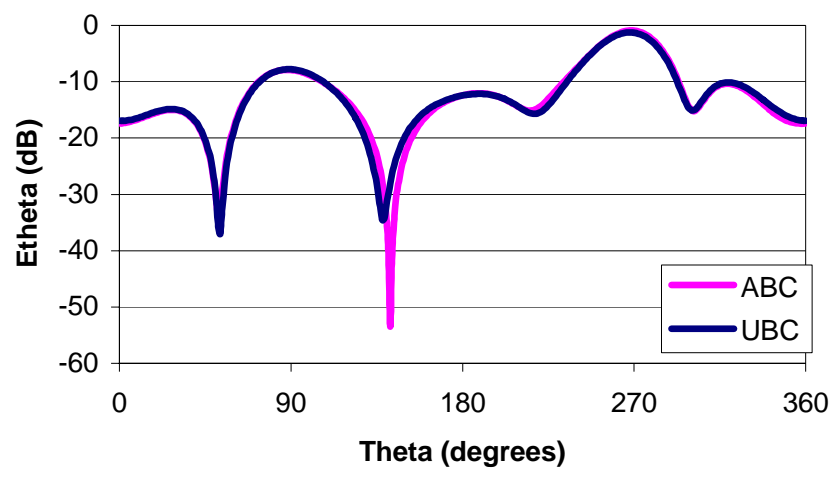

Figure 12. The $E_{\theta}$ component of the scattered electric field in the elevation plane for both the $\mathrm{ABC}$ and hybrid boundary conditions.

\section{CONCLUSION}

A hybrid Unified Boundary Condition for FEM/BEM analysis using coupled first order equations was presented and results given. The results show that that a UBC provides stability which is not found in formulations which only satisfy the $\mathrm{E}$ or $\mathrm{H}$ field on the radiating boundary. In addition, speed improvements as well as additional late-time stability were achieved by sub-cycling the BEM portion of the simulation rather than using the finite-element timestep.

\section{REFERENCES}

[1] R. Rieben, D. White, and G. Rodrigue, "High Order Symplectic Integration Methods for Finite Element Solutions to Time Dependent Maxwell Equations," IEEE Trans. Antennas Propagat., pp. 2190-2195, Aug. 2004.

[2] D. Jiao, A. Ergin, B. Shanker, E. Michielssen, J-M. Jin, “A Fast HigherOrder Time-Domain Finite Element-Boundary Integral Method for 3-D Electromagnetic Scattering Analysis," IEEE Trans. Antennas and Propagation, vol. 50, no. 9, pp. 1192-1202, Sept. 2002.

[3] R. Ziolkowski, N. Madsen, R. Carpenter, "Three-Dimensional Computer Modeling of Electromagnetic Fields: A Global Lookback Lattice Truncation Scheme," Journal of ComputationalPhysics, vol. 50, no. 3, pp 360-408, June 1983.

[4] Sarto, M. S., "Hybrid MFIE/FDTD Analysis of the Shielding Effectiveness of a Composite Enclosure Excited by a Transient Plane Wave," IEEE Transactions on Magnetics, vol. 36, no. 4, pp 946-950, July 2000.

[5] B. Shanker, M. Lu, A. Ergin, and E. Michielssen, "Plane-Wave TimeDomain Accelerated Radiation Boundary Kernels for FDTD Analysis of 3-D Electromagnetic Phenomena," IEEE Trans. Antennas and Propagation, vol. 53, no. 11, pp. 3704-3716, Nov. 2005.

[6] A. Yilmaz, Z. Lou, E. Micheilssen, J.-M. Jin, “ A Parallel time-domain adaptive integral method-accelerated single-boundary finite elementboundary element integral solver," Proc. USNC/URSI Rad. Sci. Meet., 2006, pp. 297.

[7] S. Rao, T. Sarkar, "Numerical Solution of Time Domain Integral Equations for Arbitrarily Shaped Conductor/Dielectric Composite Bodies," IEEE Trans. Antennas and Propagation, vol. 50, no. 12, pp. 1831-1837, Dec., 2002.

[8] R. Rieben, D. White, G. Rodrigue, "Improved Conditioning of Finite Element Matrices Using New High-Order Interpolatory Bases," IEEE
Trans. Antennas and Propagation, vol. 52, no. 10, pp. 2675-2683, Oct. 2004.

[9] S. Rao, D. Wilton, A. Glisson, "Electromagnetic Scattering by Surfaces of Arbitrary Shape," IEEE Trans. Antennas and Propagation, vol. 30, no. 3, pp. 409-418, May 1982. 